Research Paper

\title{
The Association between Glomerular Hyperfiltration and Left Ventricular Structure and Function in Patients with Primary Aldosteronism
}

\author{
Min-Tsun Liao', Xue-Ming Wu², Chin-Chen Chang ${ }^{3}$, Che-Wei Liao1, Ying-Hsien Chen ${ }^{4}$, Ching-Chu \\ $\mathrm{Lu}^{5}$, Yen-Ting Lin ${ }^{4}, \mathrm{Yi}-$ Yao Chang ${ }^{6}$, Chi-Sheng Hung, ${ }^{4}$ Lung-Chun Lin ${ }^{4}$, Chao-Lun Lai ${ }^{1}$, Lian-Yu Lin ${ }^{4}$, \\ Vin-Cent $\mathrm{Wu}^{4}$, Yi-Lwun $\mathrm{Ho}^{4}$, Kwan-Dun $\mathrm{Wu}^{4}$, Yen-Hung Lin ${ }^{\circledR}$, and the TAIPAI Study Group \\ 1. Department of Internal Medicine, National Taiwan University Hospital Hsin-Chu Branch, Hsin-Chu, Taiwan; \\ 2. Department of Internal Medicine, Taoyuan General Hospital, Taoyuan, Taiwan; \\ 3. Department of Medical Image, National Taiwan University Hospital and National Taiwan University College of Medicine. Taipei, Taiwan; \\ 4. Department of Internal Medicine, National Taiwan University Hospital and National Taiwan University College of Medicine. Taipei, Taiwan; \\ 5. Department of Nuclear Medicine, National Taiwan University Hospital and National Taiwan University College of Medicine. Taipei, Taiwan; \\ 6. Department of Internal Medicine, Far Eastern Memorial Hospital, New Taipei City, Taiwan
}

$\triangle$ Corresponding author: Yen-Hung Lin, MD, PhD, Division of Cardiology, Department of Internal Medicine, National Taiwan University Hospital, 7 Chung-Shan South Road, Taipei, Taiwan (e-mail: austinr34@gmail.com)

(c) 2015 Ivyspring International Publisher. Reproduction is permitted for personal, noncommercial use, provided that the article is in whole, unmodified, and properly cited. See http://ivyspring.com/terms for terms and conditions.

Received: 2014.11.03; Accepted: 2015.04.20; Published: 2015.05.03

\begin{abstract}
Background: Glomerular hyperfiltration has been recently noticed as an important issue in primary aldosteronism (PA) patients. However, its effect on the cardiovascular system remains unknown.

Methods: We prospectively analyzed 47 PA patients including 11 PA patients with estimated glomerular filtration rate (eGFR) $>130 \mathrm{ml} / \mathrm{min}$ per $1.73 \mathrm{~m}^{2}$ (group 1), and 36 PA patients with eGFR $90-110 \mathrm{ml} / \mathrm{min}$ per $1.73 \mathrm{~m}^{2}$ (group 2). Fourteen essential hypertension (EH) patients with eGFR $90-110 \mathrm{ml} / \mathrm{min}$ per $1.73 \mathrm{~m}^{2}$ were included as the control group (group 3). Echocardiography including left ventricular mass index (LVMI) measurement and tissue Doppler imaging (TDI) was performed. Predicted left ventricular mass (LVM) was calculated. Inappropriate LVM was defined as an excess of $>35 \%$ from the predicted value.

Results: The value of LVMI decreased significantly in order from groups 1 to 3 (group 1>2>3). While group 2 had a significantly higher percentage of inappropriate LVM than group 3, the percentage of inappropriate LVM were comparable in groups 1 and 2. Group 1 had a higher mitral E velocity, E/A ratio than that of group 2 . In the TDI study, the E/E' ratio also decreased significantly in order from groups 1 to 3 (group 1>2>3). Group 2 had lower E' than that of group 3, although the E' of group 1 and 2 were comparable.

Conclusions: Although PA patients with glomerular hyperfiltration were associated with higher LVMI, higher mitral E velocity, higher E/E' ratio, they had comparable E' with PA patients with normal GFR. This phenomenon may be explained by higher intravascular volume in this patient group.
\end{abstract}

Key words: Primary aldosteronism; Glomerular hyperfiltration; Left ventricular hypertrophy.

\section{Introduction}

Aldosterone as a hormone is involved in the regulation of body fluids as well as the maintenance of electrolyte balance and blood pressure (BP) homeostasis [1]. Primary aldosteronism (PA) is character- ized by the overproduction of aldosterone by the adrenal glands and is the most frequent cause of secondary hypertension [2-4]. Although the prevalence of PA was approximately $1 \%$ in patients with hyper- 
tension in older studies [5, 6], an increased prevalence of about $5-13 \%$ has been found in recent studies [4, 7], resulting from more effective methods of disease identification [8]. Long-term exposure to elevated aldosterone contributes to more cardiovascular events, such as myocardial infarction, atrial fibrillation, and left ventricle hypertrophy (LVH) independent of age, gender, and BP level [9]. Recent studies have also revealed that PA patients have significantly increased left ventricular mass (LVM) [10-12], more severe degree of diastolic function impairment [13], and myocardial fibrosis myocardial fibrosis [12, 14-16]. Activation of mineralocorticoid receptors might play a role in myocardial hypertrophy and cardiac remodeling in patients with PA [17]. The myocardial fibrosis might result from interactions of aldosterone with angiotensins, endothelin, and bradykinin[18]. Despite the direct effect of aldosterone, aldosterone also induces macrophage activation and low grade inflammation, which may play an important role in cardiac fibrosis[19]. The decrease of LVM and improvement of myocardial fibrosis after adrenalectomy [11, 14, 15] suggests that the alternation of cardiac structure is reversible (at least partially) after removal of excess aldosterone stimulation.

Glomerular hyperfiltration has been recently observed as a common phenomenon in PA patients [20-23]. A recent meta-analysis study reveals relative glomerular hyperfiltration to be the hallmark in PA and the phenomenon is beyond the effect of hypertension [24]. In patients with early stage EH, glomerular hyperfiltration indicates early target organ damage, such as LVH [25]. However, whether glomerular hyperfiltration is associated with cardiac structure or functional change in patients with PA is unclear.

The goal of this study is to evaluate the association between glomerular hyperfiltration with left ventricular (LV) structure and function in PA patients.

\section{Method \\ Patients}

This prospective study enrolled 47 PA patients including $11 \mathrm{PA}$ patients with glomerular hyperfiltration (eGFR $>130 \mathrm{ml} / \mathrm{min}$ per $1.73 \mathrm{~m}^{2}$, group 1 ) and 36 PA patients with normal eGFR $(90-110 \mathrm{ml} / \mathrm{min}$ per $1.73 \mathrm{~m}^{2}$, group 2) who were evaluated and registered in the Taiwan Primary Aldosteronism Investigation (TAIPAI) database from October 2007 to October 2010. The database was constructed for quality assurance at one medical center (National Taiwan University Hospital, Taipei, Taiwan), one branch hospital (National Taiwan University Hospital, Yun-Lin Branch, Yun-Lin, southern Taiwan) and two cooperative hospitals (Far-Eastern Memorial Hospital, Taipei;
Tao-Yuan General Hospital, Tao-Yuan, central Taiwan) $[11,14,15,26-29]$. Another $14 \mathrm{EH}$ patients with normal eGFR $\left(90-110 \mathrm{ml} / \mathrm{min}\right.$ per $\left.1.73 \mathrm{~m}^{2}\right)$ were enrolled as the control group (group 3). Body mass index (BMI) was calculated and eGFR was obtained using the Chinese Modification of Diet in Renal Disease (MDRD) Study equation $(\mathrm{eGFR}=186.0 \bullet$ [serum creatinine $]^{-1.154} \bullet$ age $^{-0.203} \bullet[0.742$ if women]) [30, 31]. The definition of renal hyperfiltration and normal eGFR were described previously $[32,33]$. The serum biochemistry studies were measured at the first evaluation of these patients at the National Taiwan University Hospital. All antihypertensive medications were discontinued for at least 21 days before measuring plasma aldosterone concentration (PAC) and plasma renin activity (PRA) levels. Diltiazem and/or doxazosin were administered for control of marked high blood pressure when required. Homeostasis Model Assessment- insulin resistance (HOMA-IR) index was calculated as insulin $(\mu \mathrm{U} / \mathrm{mL}) \times$ glucose $(\mathrm{mg} / \mathrm{dL}) / 405$. Medical histories, including demographics and medication, were carefully recorded.

\section{Diagnostic criteria of subtypes of PA}

The diagnosis of aldosterone-producing adenoma (APA) was validated by the 'modified four-corner approach', which requires all of the following criteria to be met [12, 27, 34, 35]: (1) evidence of autonomous excess aldosterone production based on an ARR (aldosterone-renin ratio) $>35$ or urine $\geq 12 \mu \mathrm{g} / 24 \mathrm{~h}$, and a TAIPAI score more than $60 \%$ [22], as well as a post-saline loading PAC (plasma aldosterone concentration) > $10 \mathrm{ng} / \mathrm{dl}$; (2) lateralization of aldosterone secretion at AVS (adrenal vein sampling) or during dexamethasone suppression adrenocortical scintigraphy (NP-59 SPECT/CT) [36]; (3) evidence of adenoma at computer tomography (CT) scan; and (4) pathologically proven adenoma after an adrenalectomy if operated, and cure of hypertension without anti-hypertensive agents or improved hypertension, potassium, PAC, and PRA (plasma renin activity) as previously described. Idiopathic hyperaldosteronism (IHA) was classified by the following criteria: (1) evidence of autonomous excess aldosterone production based on an ARR $>35$ and TAIPAI score more than $60 \%$; or urine $\geq 12 \mu \mathrm{g} / 24 \mathrm{~h}$ and post-saline loading PAC > $10 \mathrm{ng} / \mathrm{dl}$; (2) non-lateralization of aldosterone secretion at AVS or during dexamethasone suppression adrenocortical scintigraphy (NP-59 SPECT/CT) [36]; (3) evidence of bilateral diffuse enlargement on CT scan; and/or (4) evidence of diffuse cell hyperplasia in the pathology studies.

\section{Echocardiography}

A Hewlett-Packard Sonos 5500 ultrasound sys- 
tem equipped with a S3 transducer was used for the evaluation. Echocardiography was performed especially for the present study. Besides, all echocardiographic data were quantified by a trained cardiologist who was blinded to the clinical status and data of the patients. Echocardiography included two-dimensional, M-mode and Doppler ultrasound recordings. The left ventricular dimension, septum and posterior wall thickness, left atrial diameter and left ventricular ejection fraction (M-mode) were measured via the parasternal long-axis view according to the procedures of the American Society of Echocardiography. The left ventricular mass index (LVMI) was calculated according to the method of Devereux et al. [37]. LVH was defined as LVMI $\geq 134 \mathrm{gm}^{-2}$ in men and $\geq 110 \mathrm{gm}^{-2}$ in women [38]. One additional index of LV concentric geometry is end-diastolic relative wall thickness (RWT), which allows further classification of LV mass increase. RWT is defined as the ratio of posterior wall thickness to one half of left ventricular end-diastolic diameter. In patients with LVH, a RWT of more than 0.42 has been used as a threshold of concentric LVH and less than 0.42 as eccentric LVH. In patients with normal LVMI, a RWT of more than 0.42 has been used as a threshold of concentric remodeling and less than 0.42 as normal geometry [39]. LV end-diastolic and end-systolic volumes were calculated with the Teichholz method [40].

The theoretical value of predicted LVM was estimated using an equation developed previously: predicted LVM $(\mathrm{pLVM})=55.37+66.4 \times$ height $\left(\mathrm{m}^{2.7}\right)+0.64 \times$ Stroke work $(\mathrm{SW})-18.07 \times$ gender ( where gender was coded as male $=1$ and female $=2$ ). Stroke work was calculated as systolic blood pressure (SBP) (in $\mathrm{mmHg}$ ) x stroke volume $x 0.0144$ [41]. Inappropriate LVM was defined as an excess of $>35 \%$ from the predicted value [41].

Pulsed wave Doppler echocardiography for the left ventricular diastolic mitral flow was performed from the apical 4-chamber view with a 3-mm sample volume at the tip of the mitral leaflets. Transmitral flow velocity with Doppler was performed in the apical 4-chamber view, with E velocity, A velocity and mitral E-wave deceleration time being measured.

The mitral annular velocities were obtained by tissue Doppler imaging. From the apical 4-chamber view, we replaced the $3-\mathrm{mm}$ sample volume at the septal and lateral margins of the mitral annulus. Doppler samples were obtained at end-expiration during normal respiration. We measured the average of early diastole $\left(\mathrm{E}^{\prime}\right)$ velocity and late diastole $\left(\mathrm{A}^{\prime}\right)$ velocity at septal and lateral mitral annulus. The ratio of transmitral Doppler early filling velocity to tissue Doppler early diastolic mitral annular velocity (E/ $E^{\prime}$ ratio) was also calculated. At least three cycles were analyzed. Inter- and intraobserver studies were available according to our echocardiography lab. The intraobserver variability of mean mitral $\mathrm{E}^{\prime}$ was $1.67 \%$ and the interobserver variability of mean mitral $\mathrm{E}^{\prime}$ was $2.38 \%$.[42]

\section{Statistical analysis}

Data were expressed as mean \pm SD. The $t$ test was used to compare continuous data between the two groups. Differences between proportions were assessed with the chi-square test or Fisher exact test. Pearson's correlation test was used to analyze the association between LVMI and its determinants. Data of PRA, ARR were log-transformed due to non-normality which was tested by the Kolmogorov-Smirnov test. Significant determinants in the Pearson's correlation test $(p<0.05)$ were then tested with a multivariate linear regression test with stepwise subset selection to identify independent factors associated with LVMI or E/ E'.

In the present study, the mean LVMI was 156.73 $\pm 26.80 \mathrm{~g} / \mathrm{m}^{2}$ in group 1 patients. In our previous study, the mean LVMI in essential hypertensive patients was $102 \pm 22 \mathrm{~g} / \mathrm{m}^{2}$.[13] Assuming that the correlation between LVMI in two groups was 0, the standard deviation of difference of mean LVMI between two groups was 34.673, and the sample size in group 1 patients was 11, a two group t-test with a 0.05 two-sided significance level would have $80 \%$ power to detect the difference between two groups when the sample sizes in the EH patients (control group) was 6. We recruited 14 subjects of $\mathrm{EH}$ as the control group to achieve a power of more than $95 \%$.

The variables that were included as potential association of LVMI in the multivariate linear regression test for all patients (groups 1, 2 and 3) were APA, eGFR, SBP, diastolic blood pressure (DBP), mean blood pressure (MBP), serum potassium level, usage of $\alpha$-blocker and usage of $\beta$-blocker. The variables that were included as potential association of LVMI in a multivariate linear regression test for $\mathrm{PA}$ patients (groups 1 and 2) were eGFR, SBP, DBP, MBP, serum potassium level and usage of $\beta$-blocker. The variables that were included as potential association of $E / E^{\prime}$ ratio in the multivariate linear regression test for all patients (groups 1, 2 and 3) were age, body height, $\mathrm{BMI}$, serum potassium level, usage of spironolactone, APA, eGFR, SBP, DBP and MBP. The variables that were included as potential association of $E / E^{\prime}$ ratio in a multivariate linear regression test for PA patients (groups 1 and 2) were age, body height, BMI, serum potassium level, usage of spironolactone, eGFR, SBP, MBP and usage of $\beta$-blocker. Statistical analyses were performed with SPSS version 18.0 for Windows (SPSS Inc, Chicago, IL, USA). A $p$ value $<0.05$ was consid- 
ered to indicate statistical significance.

\section{Results}

\section{Patient characteristics}

Sixty-one patients were enrolled including 11 PA patients with eGFR $>130 \mathrm{ml} / \mathrm{min}$ per $1.73 \mathrm{~m}^{2}$ (group 1), 36 PA patients with eGFR $90-110 \mathrm{ml} / \mathrm{min}$ per 1.73 $\mathrm{m}^{2}$ (group 2) and $14 \mathrm{EH}$ patients with eGFR 90-110 $\mathrm{ml} / \mathrm{min}$ per $1.73 \mathrm{~m}^{2}$ (group 3). The clinical data are shown in Table 1. Patients with PA (groups 1 and 2) had significantly lower serum potassium than that of patients with EH (group 3). Among the PA patients, group 1 patients had significantly lower serum potassium than group 2 patients'.

Group 1 patients had significantly lower body height $(\mathrm{P}=0.022)$ than group 3 patients'. For medication usage, the percentage of PA patients (groups 1 and 2) using spironolactone was higher than that of $\mathrm{EH}$ patients (group 3), and the percentage of group 1 patients using a-blocker was higher than that of group 3.

\section{Echocardiographic data}

In echocardiographic measurement (Table 2), PA patients (groups 1 and 2) patients had significantly higher LVMI than EH patients' (group 3). Among the PA patients, group 1 patients had significantly higher LVMI than group 2 patients'. Group 2 patients had a higher percentage of inappropriate LVM than group 3 patients' $(\mathrm{P}=0.024)$. The percentage of inappropriate LVM was similar for groups 1 and $2(\mathrm{P}=0.740)$.

In a conventional Doppler analysis, group 1 patients had higher E velocity and a higher E/A ratio than those of group 2 patients. The two groups had similar A velocity and mitral E-wave deceleration times. Group 2 and group 3 had similar conventional Doppler parameters. In the TDI study, the PA patients (groups 1 and 2) had significantly higher $E / E^{\prime}$ ratio than was the case for $\mathrm{EH}$ patients (group 3). Among the PA patients, group 1 patients had had significantly higher $\mathrm{E} / \mathrm{E}^{\prime}$ ratio than was the case for group 2 patients.

In the factor analysis of LVMI in all patients, eGFR showed a significantly positive association with LVMI $(\mathrm{P}=0.002)$, and serum potassium levels showed a significantly negative association with LVMI $(\mathrm{P}=$ 0.001). Other significant factors associated with LVMI included SBP, DBP, MBP, a-blocker, $\beta$-blocker and presence of APA (Table 3). In the multivariate analysis of LVMI in all patients, eGFR $(\mathrm{P}=0.020), \mathrm{MBP}(\mathrm{P}=$ $0.001)$ and APA $(P=0.010)$ were independent factors associated with LVMI (Table 5).

In the factor analysis of LVMI in PA patients (groups 1 and 2), eGFR showed a significantly positive association with LVMI $(\mathrm{P}=0.009)$ and serum po- tassium levels showed a significantly negative association with LVMI $(P=0.008)$. Other significant factors associated with LVMI included SBP, DBP, MBP and $\beta$-blocker (Table 4). In the multivariate analysis of LVMI in PA patients (groups 1 and 2), eGFR (P = $0.055)$ and MBP $(\mathrm{P}=0.003)$ were independent factors associated with LVMI (Table 6). And eGFR showed a significantly positive association with predicted LVM $(\mathrm{P}=0.012)$, but was not correlated with inappropriate $\operatorname{LVM}(\mathrm{P}=0.998)$ (not shown in table). The prevalence of concentric remodeling was $0 \%$ in group $1,33 \%$ in group 2 and $36 \%$ in group 3 . It was significant between the group 1 and group 3 . $(\mathrm{P}<0.05$, table 2$)$ Group 1 had more concentric LVH ( $82 \%$ vs $45 \%)$ and less concentric remodeling ( $0 \%$ vs 33\%) than group 2 (both $\mathrm{P}<0.05$ ). (Table 2)

Table 1. Baseline Characteristics of the Study Population

\begin{tabular}{|c|c|c|c|c|}
\hline \multirow[t]{2}{*}{ Clinical characteristics } & \multirow{2}{*}{$\begin{array}{l}\text { Group } 1 \\
\mathrm{~N}=11\end{array}$} & \multirow{2}{*}{$\begin{array}{l}\text { Group } 2 \\
\mathrm{~N}=36\end{array}$} & \multicolumn{2}{|l|}{ Group 3} \\
\hline & & & $\mathrm{N}=14$ & Pvalue \\
\hline Age, y & $43 \pm 12$ & $49 \pm 13$ & $48 \pm 16$ & 0.454 \\
\hline Sex (male) & $4(36)$ & $15(42)$ & $6(43)$ & 0.978 \\
\hline Diabetes Mellitus & 0 & $5(14)$ & 0 & 0.151 \\
\hline Prior myocardial infarction & 0 & 0 & 0 & - \\
\hline Coronary artery disease & 0 & $2(6)$ & 1 & 0.688 \\
\hline Stroke & 0 & $5(14)$ & 1 & 0.371 \\
\hline Heart failure & 0 & 0 & 0 & - \\
\hline Peripheral artery disease & 0 & 0 & 0 & - \\
\hline Body weight, $\mathrm{kg}$ & $65 \pm 12$ & $67 \pm 15$ & $65 \pm 13$ & 0.937 \\
\hline Body height, $\mathrm{cm}$ & $157 \pm 8^{*}$ & $162 \pm 8$ & $165 \pm 9$ & 0.048 \\
\hline Body mass index, $\mathrm{kg} / \mathrm{m}^{2}$ & $26.5 \pm 3.6$ & $25.2 \pm 4.1$ & $23.5 \pm 2.5$ & 0.135 \\
\hline APA & $9(82)$ & $29(81)$ & $0(0)$ & $<0.001$ \\
\hline Microalbumiuria & $5(45)$ & $8(22)$ & $1(7)$ & 0.077 \\
\hline $\mathrm{SBP}, \mathrm{mmHg}$ & $169 \pm 23$ ฯ & $151 \pm 22$ & $150 \pm 22$ & 0.060 \\
\hline $\mathrm{DBP}, \mathrm{mmHg}$ & $97 \pm 12$ & $93 \pm 12$ & $91 \pm 12$ & 0.503 \\
\hline $\mathrm{MBP}, \mathrm{mmHg}$ & $121 \pm 16$ & $112 \pm 14$ & $111 \pm 14$ & 0.184 \\
\hline $\begin{array}{l}\text { Estimate duration of hyper- } \\
\text { tension, } y \\
\text { Laboratory variables }\end{array}$ & $3.8 \pm 3.2$ & $6.4 \pm 8.2$ & $4.8 \pm 4.6$ & 0.490 \\
\hline Fasting glucose, $\mathrm{mg} / \mathrm{dL}$ & $85.6 \pm 6.9$ & $93.9 \pm 14.7$ & $88.9 \pm 7.9$ & 0.116 \\
\hline Insulin, $\mu \mathrm{U} / \mathrm{mL}$ & $11.7 \pm 15.3$ & $15.8 \pm 15.9$ & $7.8 \pm 2.7$ & 0.290 \\
\hline HOMA-IR, mU/L & $2.5 \pm 3.3$ & $3.8 \pm 3.8$ & $1.7 \pm 0.6$ & 0.220 \\
\hline Creatinine, mg/dL & $0.72 \pm 0.12^{\S}, \Psi$ & $0.93 \pm 0.14$ & $0.93 \pm 0.15$ & $<0.001$ \\
\hline Potassium, mmol/L & $3.1 \pm 0.6 \square$ & $3.7 \pm 0.6^{\Delta}$ & $4.1 \pm 0.3$ & $<0.001$ \\
\hline $\mathrm{PAC}, \mathrm{ng} / \mathrm{dL}$ & $52 \pm 34$ & $50 \pm 32$ & $29 \pm 15$ & 0.072 \\
\hline PRA, ng/ml/hr & $0.25 \pm 0.23^{\Psi}$ & $1.1 \pm 2.3^{\Delta}$ & $9.7 \pm 9.0$ & $<0.001$ \\
\hline ARR & $2481 \pm 4166^{*}$ & $1461 \pm 3171$ & $11 \pm 13$ & 0.121 \\
\hline Log PRA & $-1.00 \pm 0.76 \square$ & $-0.051 \pm 0.86$ 羊 & $0.72 \pm 0.54$ & $<0.001$ \\
\hline Log ARR & $2.63 \pm 0.92 \square$ & $2.13 \pm 0.98 \stackrel{¥}{¥}$ & $0.69 \pm 0.61$ & $<0.001$ \\
\hline \multicolumn{5}{|l|}{ Hypertension medication } \\
\hline $\mathrm{CCB}$ & $7(64)$ & $14(39)$ & $7(50)$ & 0.380 \\
\hline ACEI/ARB & $3(37)$ & $13(36)$ & $9(64)$ & 0.114 \\
\hline Spironolactone & $6(55)^{\Psi}$ & $16(44)^{\Delta}$ & $0(0)$ & 0.002 \\
\hline a-blocker & $7(64)^{\Psi}$ & $11(31)$ & $1(7)$ & 0.013 \\
\hline$\beta$-blocker & $3(27)$ & $16(44)$ & $5(36)$ & 0.628 \\
\hline
\end{tabular}

Values are mean \pm SD. APA: aldosterone-producing adenoma; SBP: systolic blood pressure; DBP: diastolic blood pressure; MBP: mean blood pressure; PAC: plasma aldosterone concentration; PRA: plasma renin activity; ARR: aldosterone-renin ratio; CCB: calcium channel blocker; ACEI: angiotensin-converting enzyme inhibitor; ARB: angiotensin receptor blocker; HOMA-IR: homeostatic model assessment-insulin resistance

${ }^{*} P<0.05$ between groups 1 and $3 ; \uparrow P<0.05$ between groups 1 and $2 ; s P<0.001$ between groups 1 and $2 ; \square<0.001$ between groups 1 and $3 ; \square<0.01$ between groups 1 and $2{ }^{\wedge} P<$ 0.01 between groups 2 and 3 ; ${ }^{\Psi} P<0.01$ between groups 1 and $3 ;{ }^{*} P<0.001$ between groups 2 and 3 . 
Table 2. Baseline Echocardiographic Parameters of the Study Population

\begin{tabular}{|c|c|c|c|c|}
\hline \multirow{2}{*}{$\begin{array}{l}\text { Echocardiographic } \\
\text { parameters }\end{array}$} & \multirow{2}{*}{$\begin{array}{l}\text { Group } 1 \\
\mathrm{~N}=11\end{array}$} & \multirow{2}{*}{$\begin{array}{l}\text { Group } 2 \\
\mathrm{~N}=36\end{array}$} & \multicolumn{2}{|l|}{ Group 3} \\
\hline & & & $\mathrm{N}=14$ & $\begin{array}{l}\mathrm{P} \\
\text { value }\end{array}$ \\
\hline IVST, mm & $12.09 \pm 1.51$ & $11.75 \pm 2.26$ & $10.64 \pm 1.60$ & 0.145 \\
\hline LVPWT, mm & $12.09 \pm 1.64 \square$ & $11.00 \pm 2.06^{\Omega}$ & $9.57 \pm 1.34$ & 0.005 \\
\hline LVEDD, mm & $47.27 \pm 4.38$ & $45.08 \pm 4.88$ & $46.00 \pm 4.85$ & 0.405 \\
\hline LVESD, mm & $28.36 \pm 3.14$ & $28.39 \pm 4.65$ & $27.79 \pm 5.85$ & 0.545 \\
\hline Relative wall thickness & $0.52 \pm 0.08$ & $0.51 \pm 0.11^{\Omega}$ & $0.44 \pm 0.06$ & 0.066 \\
\hline LVMI, $\mathrm{g} / \mathrm{m}^{2}$ & $156.73 \pm 26.804, \square$ & $127.05 \pm 33.87^{\Omega}$ & $109.78 \pm 23.61$ & 0.001 \\
\hline LVH & $11 / 11(100)^{\square \Psi}$ & $21 / 36(58)$ & $4 / 14(29)$ & 0.001 \\
\hline Concentric LVH & $9 / 11(82)^{*}$ & $17 / 36(45)$ & $4 / 14(29)$ & 0.026 \\
\hline Eccentric LVH & $2 / 11(18)$ & $4 / 36(11)$ & $0 / 14(0)$ & 0.343 \\
\hline Concentric remodeling & $0 / 11(0)^{*}$, & $12 / 36(33)$ & $5 / 14(36)$ & 0.053 \\
\hline Normal Geometry & $0 / 11(0)^{*}$ & $3 / 36(8)^{\Omega}$ & $5 / 14(36)$ & 0.019 \\
\hline LVM, gm & $258.96 \pm 61.78^{\Psi}$ & $220.67 \pm 74.69$ & $190.27 \pm 54.05$ & 0.052 \\
\hline Predicted LV mass, gm & $184.22 \pm 47.64$ & $155.74 \pm 39.17$ & $167.93 \pm 49.70$ & 0.157 \\
\hline $\begin{array}{l}\text { Observed/predicted } \\
\operatorname{LVM}(\%)\end{array}$ & $143.20 \pm 29.63^{*}$ & $143.42 \pm 34.68^{\Delta}$ & $114.87 \pm 22.21$ & 0.016 \\
\hline Inappropriate LVM & $5 / 11(45)$ & $19 / 36(53)^{\Omega}$ & 2/14 (14) & 0.044 \\
\hline LVEDV & $104.93 \pm 22.71$ & $94.40 \pm 23.64$ & $98.80 \pm 24.13$ & 0.422 \\
\hline LVESV & $31.07 \pm 8.27$ & $31.92 \pm 15.44$ & $28.46 \pm 14.23$ & 0.742 \\
\hline LVEF, \% & $69.82 \pm 5.15$ & $66.08 \pm 8.44$ & $71.79 \pm 9.59$ & 0.075 \\
\hline $\mathrm{E}, \mathrm{cm} / \mathrm{s}$ & $85.82 \pm 19.10 \pi$ & $71.08 \pm 15.04$ & $72.29 \pm 14.95$ & 0.029 \\
\hline $\mathrm{A}, \mathrm{cm} / \mathrm{s}$ & $68.64 \pm 15.15$ & $75.17 \pm 20.29$ & $67.21 \pm 16.78$ & 0.325 \\
\hline E/A ratio & $1.32 \pm 0.42^{\star}$ & $0.98 \pm 0.27$ & $1.12 \pm 0.18$ & 0.005 \\
\hline DT, sec & $181.36 \pm 36.49$ & $180.86 \pm 44.78$ & $188.36 \pm 38.54$ & 0.847 \\
\hline \multicolumn{5}{|l|}{ Tissue doppler } \\
\hline Mean $E^{\prime}, \mathrm{cm} / \mathrm{s}$ & $8.05 \pm 3.31^{*}$ & $7.78 \pm 2.05^{\Delta}$ & $10.13 \pm 1.86$ & 0.008 \\
\hline Mean $A^{\prime}, \mathrm{cm} / \mathrm{s}$ & $10.65 \pm 2.07$ & $11.49 \pm 2.43$ & $11.03 \pm 1.81$ & 0.522 \\
\hline Mean E/E' ratio & $12.57 \pm 5.72^{\natural, ~} \square$ & $9.74 \pm 2.60^{\Omega}$ & $7.51 \pm 2.16$ & 0.002 \\
\hline \multicolumn{5}{|c|}{$\begin{array}{l}\text { Value are mean } \pm \text { SEM. IVST= interventricular septal thickness; } L V P W T=\text { left ventricular } \\
\text { posterior wall thickness; } L V E D D=\text { left ventricular end-diastolic diameter; } L V E S D=\text { left } \\
\text { ventricular end-systolic diameter; } L V M I=\text { left ventricular mass index; } L V H=\text { left ventric- } \\
\text { ular hypertrophy; } L V M=\text { left ventricular mass; } L V E D V=\text { left ventricular end-diastolic } \\
\text { volume; } L V E S V=\text { left ventricular end-systolic volume; } L V E F=l \text { eft ventricular ejection } \\
\text { fraction; DT=deceleration time }\end{array}$} \\
\hline
\end{tabular}

Table 3. Factors associated with LVMI (all patients, $n=61$ )

\begin{tabular}{lll}
\hline & Person correlation coefficient & P value \\
\hline Age, y & -0.193 & 0.137 \\
Sex (male) & 0.031 & 0.812 \\
Body weight, kg & 0.132 & 0.312 \\
Body height, cm & 0.116 & 0.372 \\
Body mass index, kg/m² & 0.120 & 0.357 \\
APA & 0.382 & 0.003 \\
HOMA-IR & -0.137 & 0.363 \\
Presence of Diabetes Mellitus & -0.137 & 0.366 \\
Presence of Microalbumiuria & 0.155 & 0.232 \\
eGFR, ml/min per 1.73 m & 0.383 & 0.002 \\
SBP, mmHg & 0.462 & $<0.001$ \\
DBP, mmHg & 0.394 & 0.002 \\
MBP, mmHg & 0.454 & $<0.001$ \\
Estimate duration of hyperten- & 0.085 & 0.516 \\
sion, y & & \\
Potassium, mmol/L & -0.422 & 0.001 \\
Presence of microalbuminuria & 0.155 & 0.232 \\
PAC, ng/dL & 0.103 & 0.440 \\
Log PRA & -0.189 & 0.156 \\
Log ARR & 0.191 & 0.151 \\
CCB & 0.071 & 0.640 \\
ACEI/ARB & -0.165 & 0.203 \\
Spirololactone & 0.077 & 0.555 \\
a-blocker & 0.270 & 0.035 \\
$\beta$-blocker & -0.275 & 0.032 \\
\hline APA:alosternepros & & \\
\hline
\end{tabular}

APA: aldosterone-producing adenoma; eGFR: estimated glomerular filtration rate; SBP: systolic blood pressure; DBP: diastolic blood pressure; MBP: mean blood pressure; PAC: plasma aldosterone concentration; PRA: plasma renin activity
Table 4. Factors associated with LVMI (PA patients, $n=47$ )

\begin{tabular}{lll}
\hline & $\begin{array}{l}\text { Person correlation } \\
\text { coefficient }\end{array}$ & P value \\
\hline Age, y & -0.185 & 0.213 \\
Sex (male) & 0.130 & 0.383 \\
Body weight, kg & 0.077 & 0.606 \\
Body height, cm & 0.166 & 0.265 \\
Body mass index, kg/m² & 0.023 & 0.878 \\
APA & 0.255 & 0.087 \\
HOMA-IR & -0.250 & 0.141 \\
Presence of Diabetes Mellitus & -0.190 & 0.202 \\
Presence of Microalbumiuria & 0.175 & 0.241 \\
eGFR, ml/min per 1.73 m² & 0.376 & 0.009 \\
SBP, mmHg & 0.491 & $<0.001$ \\
DBP, mmHg & 0.401 & 0.005 \\
MBP, mmHg & 0.473 & 0.001 \\
Estimate duration of hypertension, y & 0.083 & 0.580 \\
Potassium, mmol/L & -0.388 & 0.008 \\
PAC, ng/dL & 0.010 & 0.946 \\
Log PRA & 0.020 & 0.898 \\
Log ARR & -0.030 & 0.849 \\
CCB & 0.038 & 0.800 \\
ACEI/ ARB & -0.087 & 0.561 \\
Spirololactone & -0.058 & 0.697 \\
a-blocker & 0.204 & 0.170 \\
$\beta$-blocker & -0.444 & 0.002 \\
\hline APA: aldosterone-producing adenoma; eGFR: estimated glomerular filtration rate; SBP: \\
systolic blood pressure; DBP: diastolic blood pressure; MBP: mean blood pressure; PAC: \\
plasma aldosterone concentration; PRA: plasma renin activity; ARR: aldosterone-renin \\
ratio & & \\
& &
\end{tabular}

Table 5. Multivariate regression analysis with left ventricular mass index as the dependent variable. All patients, $n=61$, model adjusted $R^{2}=0.373$

\begin{tabular}{|c|c|c|c|}
\hline & B $(95 \% \mathrm{CI})$ & $P$ value & Tolerance \\
\hline $\mathrm{MBP}, \mathrm{mmHg}$ & $0.880(1.031 ; 0.629)$ & 0.001 & 0.960 \\
\hline APA & $20.279(27.883 ; 12.675)$ & 0.010 & 0.982 \\
\hline eGFR, $\mathrm{ml} / \mathrm{min}$ per $1.73 \mathrm{~m}^{2}$ & $0.496(0.702 ; 0.290)$ & 0.020 & 0.945 \\
\hline
\end{tabular}

Table 6. Multivariate regression analysis with left ventricular mass index as the dependent variable. PA patients, $n=47$, model adjusted $R^{2}=0.303$

\begin{tabular}{llll}
\hline & $B(95 \% \mathrm{CI})$ & $P$ value & Tolerance \\
\hline MBP, mmHg & $0.958(1.261 ; 0.655)$ & 0.003 & 0.927 \\
eGFR, ml $/$ min per $1.73 \mathrm{~m}^{2}$ & $0.455(0.686 ; 0.224)$ & 0.055 & 0.927 \\
\hline
\end{tabular}

MBP: mean blood pressure; eGFR: estimate glomerular filtration rate; excluded variables: systolic blood pressure, diastolic blood pressure, serum potassium level, and usage of $\beta$-blocker

In the factor analysis of $\mathrm{E} / \mathrm{E}^{\prime}$ ratio in all patients, eGFR showed a significantly positive association with $\mathrm{E} / \mathrm{E}^{\prime}$ ratio $(\mathrm{P}=0.007$, not shown in the table). Other significant factors associated with $\mathrm{E} / \mathrm{E}^{\prime}$ ratio included PA, age, body height, BMI, SBP, serum potassium level, spironolactone, a-blocker and $\beta$-blocker. In the multivariate analysis of $\mathrm{E} / \mathrm{E}^{\prime}$ ratio in all patients, eGFR $(P=0.001), \beta$-blocker $(p=0.007)$, PA $(p=0.021)$ and age $(p=0.022)$ were independent factors associated with $\mathrm{E} / \mathrm{E}^{\prime}$ ratio (Table 7). 
In the factor analysis of $\mathrm{E} / \mathrm{E}^{\prime}$ ratio in PA patients (groups 1 and 2), eGFR showed a significantly positive association with $\mathrm{E} / \mathrm{E}^{\prime}$ ratio $(\mathrm{P}=0.037$, not shown in the table). Other significant factors associated with $\mathrm{E} / \mathrm{E}^{\prime}$ ratio included body height, BMI, SBP, MBP and $\beta$-blocker. In the multivariate analysis of $E / E^{\prime}$ ratio in PA patients (groups 1 and 2), eGFR $(\mathrm{P}=0.003)$ and $\beta$-blocker $(\mathrm{P}=0.001)$ were independent factors associated with $\mathrm{E} / \mathrm{E}^{\prime}$ ratio (Table 8 ).

Table 7. Multivariate regression analysis with E/E' ratio as the dependent variable. All patients, $n=61$, model adjusted $R^{2}=0.408$

\begin{tabular}{llll}
\hline & $B(95 \% \mathrm{CI})$ & P value & Tolerance \\
\hline eGFR, ml/min per $1.73 \mathrm{~m}^{2}$ & $0.081(0.058 ; 0.104)$ & 0.001 & 0.914 \\
Usage of $\beta$-blocker & $2.413(1.558 ; 3.268)$ & 0.007 & 0.908 \\
PA & $2.276(1.320 ; 3.232)$ & 0.021 & 0.951 \\
Age & $0.075(0.053 ; 0.107)$ & 0.022 & 0.910
\end{tabular}

eGFR: estimate glomerular filtration rate; PA: primary aldosteronism; excluded variables: body height, body mass index, serum potassium level, systolic blood pressure, diastolic blood pressure, mean blood pressure, presence of aldosterone-producing adenoma, and usage of spironolactone

Table 8. Multivariate regression analysis with E/E' ratio as the dependent variable. $P A$ patients, $n=47$, model adjusted $R^{2}=0.309$

\begin{tabular}{llll}
\hline & $\mathcal{B}(95 \% \mathrm{CI})$ & P value & Tolerance \\
\hline eGFR, $\mathrm{ml} /$ min per $1.73 \mathrm{~m}^{2}$ & $0.079(0.054 ; 0.104)$ & 0.003 & 0.952 \\
Usage of $\beta$-blocker & $3.552(2.544 ; 4.560)$ & 0.001 & 0.952 \\
\hline
\end{tabular}

eGFR: estimate glomerular filtration rate; excluded variables: age, body height, body mass index, serum potassium level, usage of spironolactone, systolic blood pressure, and mean blood pressure

\section{Discussion}

The major findings of this study are as follows: (1) PA patients had a higher LVMI and worse diastolic function compared to EH patients; (2) PA patients with glomerular hyperfiltration had a higher LVMI than that of PA patients with normal eGFR, which is majorly contributed by concentric $\mathrm{LVH}$ and predicted LVM; (3) PA patients with glomerular hyperfiltration had higher $\mathrm{E} / \mathrm{E}^{\prime}$ ratio than but comparable $\mathrm{E}^{\prime}$ to $\mathrm{PA}$ patients with normal eGFR, which is majorly contributed by increased E velocity. This is the first human study to demonstrate the association between glomerular hyperfiltration and other target organ damage such as cardiac structure or function change in PA patients.

EH patient had more normal LV geometry than PA patients with normal eGFR. PA patients with normal eGFR had more concentric LVH and concentric remodeling than $\mathrm{EH}$ patients. And PA patients with hyperfiltration had higher rate of concentric $\mathrm{LVH}$ not concentric remodeling than PA patients with normal eGFR. We propose that hyperfiltration had an additional effect in concentric LVH beyond PA.

It is notable that PA patients had a higher ob- served-to-predicted LVM ratio and a higher percentage of "inappropriate" LVM than that of EH patients. This finding is the same as in Muiesan et al. [43]. However, among PA patients, although PA patients with hyperfiltration had a higher LVMI than PA patients with normal eGFR, the "inappropriate" LVM was similar between the two groups. Moreover, the observed-to-predicted LVM ratio was almost the same in these two groups, which means that the difference of LVM between the two groups contributed to the predicted LVM. The actual predicted LVM difference between the two groups was around $17 \%$. The predicted LVM was estimated using body height, gender, BP, and stroke volume. The $4.9 \%$ higher LVEDD (left ventricular end-diastolic diameter), 11\% higher LVEDV (left ventricular end-diastolic volume) and $12 \%$ higher SBP of group 1 patients over group 2 patients were the major contributing factors of this phenomenon. Combining the finding of higher LVEDD and LVEDV with the higher mitral E velocity of group 1 patients over group 2 patients, we can propose that the intravascular volume is higher in group 1 than in group 2.

The phenomenon of glomerular hyperfiltration in PA was first noted in 1996 [44]. Although the mechanisms for this are not fully understood, it may be attributed to enhanced tubular sodium reabsorption [44]. In a recent study by Fu et al., aldosterone activated mineralocorticoid receptors in macula densa cells were found to further increase nitric oxide production in the macula densa and to blunt the tubuloglomerular feedback response in rats [45]. This effect happens quickly and made possible through the rapid nongenomic pathway of aldosterone. Furthermore, although the influence of glomerular hyperfiltration in PA is still unclear clinically, in hypertensive patients, the relationship between glomerular hyperfiltration and microalbuminuria has been validated [46], and microalbuminuria may further cause cardiovascular and renal events [47]. In this investigation, glomerular hyperfiltration was found to be associated with increased LVMI, with LVH being recognized as a marker of hypertension-related target organ damage and being associated with a greater risk of cardiovascular events and death $[48,49]$. This present study implies a greater cardiovascular morbidity in PA patients with glomerular hyperfiltration than that of the PA patients with normal eGFR.

In this study, we found an independent association of glomerular hyperfiltration with LVMI in PA patients. Although glomerular hyperfiltration may also be a sign of more severe hyperaldosteronism, the associations among glomerular hyperfiltration and LVMI are still significant after adjustment for BP, serum potassium levels and a-blocker usage in the 
multivariate analysis. Glomerular hyperfiltration is also associated with $\mathrm{LVH}$ in patients with $\mathrm{EH}$. At a similar age, BMI, body surface area, and BP, hypertensive patients with $\mathrm{LVH}$ have been observed to have a higher glomerular filtration rate and filtration fraction than those without LVH, whereas renal blood flow and renal vascular resistance measurements were not found to be significantly different [25]. However, future studies will therefore need to investigate the possible mechanisms of how glomerular hyperfiltration can cause cardiac hypertrophy in PA patients.

In this study, we used conventional Doppler and TDI to evaluate cardiac diastolic function. We found that PA patients with glomerular hyperfiltration (group 1) had a higher E and E/A than PA patients with normal eGFR (group 2). In the TDI analysis, group 1 patients had a higher E/E' ratio than was the case for group 2. In the current study, results of the conventional echocardiography and TDI both indicate that glomerular hyperfiltration may influence the cardiac diastolic function in PA patients. The difference in $E / E^{\prime}$ ratio was due to the elevation of $E$ in PA patients with glomerular hyperfiltration. In additions, the $\mathrm{E}^{\prime}$ was similar for groups 1 and 2, which implies that the higher $\mathrm{E} / \mathrm{E}^{\prime}$ ratio in group 1 was caused by the elevated $E$ velocity $(P=0.011)$, with the elevated $\mathrm{E}$ velocity meaning early filling peak velocity, which is relative to volume status and not the LV myocardial relaxation. However, while this study was underpowered to assess a significant difference of LV internal diameter and volume between PA patients with normal eGFR and glomerular hyperfiltration, the PA patients with glomerular hyperfiltration tended to have an increased LVEDD and LVEDV. Group 2 and 3 patients had similar conventional Doppler parameters. In the TDI analysis, PA patients with normal eGFR had lower $E^{\prime}$ and higher $E / E^{\prime}$ ratio than $E H$ patients with normal eGFR. However, it has recently been demonstrated that the $\mathrm{E} / \mathrm{E}^{\prime}$ ratio of transmitral flow to mitral annulus velocity is a strong and independent association of cardiac outcome [50] and TDI is superior to conventional Doppler in evaluating diastolic function [51]. In the present study, PA patients exhibited a greater degree of diastolic dysfunction than the EH patients. Also, PA patients with glomerular hyperfiltration had a higher $\mathrm{E} / \mathrm{E}^{\prime}$ ratio than $\mathrm{PA}$ patients with normal eGFR, which may also indicate a higher degree of diastolic dysfunction. The associations among glomerular hyperfiltration and $\mathrm{E} / \mathrm{E}^{\prime}$ ratio in PA patients are significant after adjustment for body height, BMI, SBP, MBP and $\beta$-blocker usage in the multivariate analysis. However, the higher E/ $\mathrm{E}^{\prime}$ ratio in group 1 was caused by the elevated $E$ velocity, and not caused by the decreased $E^{\prime}$. The actual physi- ological meaning of the higher $\mathrm{E} / \mathrm{E}^{\prime}$ ratio in group 1 compared to group 2 needs further study.

There are limitations to this study. First, this is a cross-sectional correlation study and only shows the association between glomerular hyperfiltration and cardiac structure and functional change. Whether glomerular hyperfiltration is a factor influencing LVMI or only a marker to present more severe disease cannot be clearly elucidated in this study; that is, under this study design, we cannot establish a direct causal effect between glomerular hyperfiltration and LVMI. Second, this study only provides the data of glomerular hyperfiltration and LVMI, but the effect on cardiovascular mortality or morbidity cannot be known. Further long-term follow-up studies are needed to investigate the clinical impact of glomerular hyperfiltration on cardiovascular outcome. Third, the patient number is small in this study, especially in group 1, which may indicate a lack of power to demonstrate the difference between groups, such as the predicted LVM, LVEDD or LVEDV between groups 1 and 2. This study cannot provide the final evidence of association. On the other hand, the anti-hypertensive medications were not standard in this study and it may be hard to adjust the bias of different medications because of the small size. For example, usage of spironolactone in PA is able to improve the kidney damage and glomerular hyperfiltration [21, 52]. Further studies with a larger patient number are needed. Fourth, many parameters were tested in this study, which raised an issue of multiple comparison. However, all the clinically relevant parameters showed similar tendency and direction of statistically significance. The situation is unlikely due to random error. Fifth, there are several methods of calculate eGFR. All methods are designed for detection of renal dysfunction, and none is validated for evaluation of hyperfiltration. We used Chinese MDRD in this study, because it is the best method to calculate eGFR in Chinese population, even in health population [31]. However, the eGFR calculated by Chinese MDRD is still not validated in the status of hyperfiltration. Sixth, the estimation of theoretical value of predicted LVM is done with the blood pressure values recorded in the end of echocardiographic assessment in previous study [41]. The method is not confirmed by other studies. These may limit the application and accuracy of this method. Seventh, most patients were young and hypertension durations were short in this study. Age and duration of hypertension are correlated with LVM. The result of this study may not apply in an older population.

\section{Conclusions}

In conclusion, glomerular hyperfiltration in PA 
patients was associated with higher LVMI, higher mitral $E$ velocity, higher $E / E^{\prime}$ ratio, but there was a comparable $E^{\prime}$ in PA patients with normal GFR. This phenomenon may be explained by higher intravascular volume in this patient group.

\section{Acknowledgements}

This study was supported by Ta-Tung Kidney Foundation, National Taiwan University Hospital (NTUH 103-S2347, UN103-065), Ministry of Science and Technology (NSC 102-2314-B-002 -078 -MY3, MOST 103-2220-E-002 -011), and Department of Health, Executive Yuan, R.O.C. (DOH 102-PTH10204, $\mathrm{DOH}$ 103-HO-1012). The funders had no role in study design, data collection and analysis, decision to publish, or preparation of the manuscript.

\section{Competing Interests}

The authors have declared that no competing interest exists.

\section{References}

1. Freel EM, Connell JM. Mechanisms of hypertension: the expanding role of aldosterone. Journal of the American Society of Nephrology: JASN 2004; 15: 1993-2001.

2. Gaddam KK, Pimenta E, Husain S, Calhoun DA. Aldosterone and cardiovascular disease. Curr Probl Cardiol 2009; 34: 51-84.

3. Rossi GP, Seccia TM, Pessina AC. Primary aldosteronism - part I: prevalence, screening, and selection of cases for adrenal vein sampling. J Nephrol 2008; 21: 447-54

4. Rossi GP. Prevalence and diagnosis of primary aldosteronism. Curr Hypertens Rep 2010; 12: 342-8.

5. Hiramatsu K, Yamada T, Yukimura $Y$ et al. A screening test to identify aldosterone-producing adenoma by measuring plasma renin activity. Results in hypertensive patients. Arch Intern Med 1981; 141: 1589-93.

6. Young WF, Jr. Minireview: primary aldosteronism--changing concepts in diagnosis and treatment. Endocrinology 2003; 144: 2208-13.

7. Plouin PF, Amar L, Chatellier G. Trends in the prevalence of primary aldosteronism, aldosterone-producing adenomas, and surgically correctable aldosterone-dependent hypertension. Nephrology, dialysis, transplantation official publication of the European Dialysis and Transplant Association - European Renal Association 2004; 19: 774-7.

8. Mulatero P. Increased Diagnosis of Primary Aldosteronism, Including Surgically Correctable Forms, in Centers from Five Continents. Journal of Clinical Endocrinology \& Metabolism 2004; 89: 1045-50.

9. Milliez P, Girerd X, Plouin PF et al. Evidence for an increased rate of cardiovascular events in patients with primary aldosteronism. Journal of the American College of Cardiology 2005; 45: 1243-8.

10. Matsumura $\mathrm{K}$, Fujii $\mathrm{K}$, Oniki $\mathrm{H}$ et al. Role of aldosterone in left ventricular hypertrophy in hypertension. American journal of hypertension 2006; 19: 13-8.

11. Lin $\mathrm{YH}$, Huang $\mathrm{KH}$, Lee JK et al. Factors influencing left ventricular mass regression in patients with primary aldosteronism post adrenalectomy. Journal of the renin-angiotensin-aldosterone system : JRAAS 2011; 12: 48-53.

12. Rossi GP. Excess ldosterone Is Associated With Alterations of Myocardial Texture in Primary Aldosteronism. Hypertension 2002; 40: 23-7.

13. Lin $\mathrm{YH}$, Wang $\mathrm{SM}, \mathrm{Wu} \mathrm{VC}$ et al. The association of serum potassium level with left ventricular mass in patients with primary aldosteronism. European journal of clinical investigation 2011; 41: 743-50.

14. Lin $\mathrm{YH}, \mathrm{Wu} \mathrm{XM}$, Lee $\mathrm{HH}$ et al. Adrenalectomy reverses myocardial fibrosis in patients with primary aldosteronism. J Hypertens 2012; 30: 1606-13.

15. Lin YH, Lee HH, Liu KL et al. Reversal of myocardial fibrosis in patients with unilateral hyperaldosteronism receiving adrenalectomy. Surgery 2011; 150: 526-33.

16. Lee $\mathrm{HH}$, Hung $\mathrm{CS}, \mathrm{Wu} \mathrm{XM}$ et al. Myocardial ultrasound tissue characterization of patients with primary aldosteronism. Ultrasound in medicine E biology 2013; 39: 54-61.

17. Schmidt BM, Schmieder RE. Aldosterone-induced cardiac damage: focus on blood pressure independent effects. Am J Hypertens 2003; 16: 80-6.

18. Funder J. Mineralocorticoids and cardiac fibrosis: the decade in review. Clin Exp Pharmacol Physiol 2001; 28: 1002-6.

19. Weber KT, Gerling IC, Kiani MF et al. Aldosteronism in heart failure: a proinflammatory/fibrogenic cardiac phenotype. Search for biomarkers and potential drug targets. Curr Drug Targets 2003; 4: 505-16.
20. Sechi LA, Novello M, Lapenna $R$ et al. Long-term renal outcomes in patients with primary aldosteronism. JAMA 2006; 295: 2638-45.

21. Ribstein J, Du Cailar G, Fesler P, Mimran A. Relative glomerular hyperfiltration in primary aldosteronism. Journal of the American Society of Nephrology: JASN 2005; 16: 1320-5.

22. Wu VC, Yang SY, Lin JW et al. Kidney impairment in primary aldosteronism. Clin Chim Acta 2011; 412: 1319-25.

23. Wu VC, Kuo CC, Wang SM et al. Primary aldosteronism: changes in cystatin C-based kidney filtration, proteinuria, and renal duplex indices with treatment. J Hypertens 2011; 29: 1778-86.

24. Kuo CC, $\mathrm{Wu} \mathrm{VC}$, Tsai CW, Wu KD. Relative kidney hyperfiltration in primary aldosteronism: a meta-analysis. Journal of the renin-angiotensin-aldosterone system : JRAAS 2011; 12: 113-22.

25. Schmieder RE, Messerli FH, Garavaglia G, Nunez B. Glomerular hyperfiltration indicates early target organ damage in essential hypertension. JAMA : the journal of the American Medical Association 1990; 264: 2775-80.

26. Chang LC, Huang KC, Wu YW et al. The clinical implications of blood adiponectin in cardiometabolic disorders. Journal of the Formosan Medical Association = Taiwan yi zhi 2009; 108: 353-66.

27. Wu VC, Chang HW, Liu KL et al. Primary aldosteronism: diagnostic accuracy of the losartan and captopril tests. American journal of hypertension 2009; 22: $821-7$

28. Wu VC, Chueh SC, Chang HW et al. Bilateral aldosterone-producing adenomas: differentiation from bilateral adrenal hyperplasia. QIM : monthly journal of the Association of Physicians 2008; 101: 13-22.

29. Lin YH, Lin LY, Chen A et al. Adrenalectomy improves increased carotid intima-media thickness and arterial stiffness in patients with aldosterone producing adenoma. Atherosclerosis 2012; 221: 154-9.

30. Ma YC, Zuo L, Chen JH et al. Modified glomerular filtration rate estimating equation for Chinese patients with chronic kidney disease. Journal of the American Society of Nephrology: JASN 2006; 17: 2937-44.

31. Kong X, Ma Y, Chen J et al. Evaluation of the Chronic Kidney Disease Epidemiology Collaboration equation for estimating glomerular filtration rate in the Chinese population. Nephrology, dialysis, transplantation : official publication of the European Dialysis and Transplant Association - European Renal Association 2013; 28: 641-51.

32. Vervoort G, Veldman B, Berden JH et al. Glomerular hyperfiltration in type 1 diabetes mellitus results from primary changes in proximal tubular sodium handling without changes in volume expansion. Eur J Clin Invest 2005; 35: 330-6.

33. Cheng TY, Wen SF, Astor BC et al. Mortality risks for all causes and cardiovascular diseases and reduced GFR in a middle-aged working population in Taiwan. American journal of kidney diseases : the official journal of the National Kidney Foundation 2008; 52: 1051-60.

34. Watt GC, Harrap SB, Foy CJ et al. Abnormalities of glucocorticoid metabolism and the renin-angiotensin system: a four-corners approach to the identification of genetic determinants of blood pressure. Journal of hypertension 1992; 10: 473-82.

35. Wu VC, Chueh SC, Chang HW et al. Association of kidney function with residual hypertension after treatment of aldosterone-producing adenoma. American journal of kidney diseases : the official journal of the National Kidney Foundation 2009; 54: 665-73.

36. Yen RF, Wu VC, Liu KL et al. 131I-6beta-iodomethyl-19-norcholesterol SPECT/CT for primary aldosteronism patients with inconclusive adrenal venous sampling and CT results. J Nucl Med 2009; 50: 1631-7.

37. Devereux RB, Reichek N. Echocardiographic determination of left ventricular mass in man. Anatomic validation of the method. Circulation 1977; 55: 613-8.

38. Ayus JC, Go AS, Valderrabano F et al. Effects of erythropoietin on left ventricular hypertrophy in adults with severe chronic renal failure and hemoglobin <10 g/dL. Kidney Int 2005; 68: 788-95.

39. Lang RM, Bierig M, Devereux RB et al. Recommendations for chamber quantification: a report from the American Society of Echocardiography's Guidelines and Standards Committee and the Chamber Quantification Writing Group, developed in conjunction with the European Association of Echocardiography, a branch of the European Society of Cardiology. J Am Soc Echocardiogr 2005; 18: 1440-63.

40. Teichholz LE, Kreulen T, Herman MV, Gorlin R. Problems in echocardiographic volume determinations: echocardiographic-angiographic correlations in the presence of absence of asynergy. Am I Cardiol 1976; 37: 7-11.

41. de Simone G, Devereux RB, Kimball TR et al. Interaction between body size and cardiac workload: influence on left ventricular mass during body growth and adulthood. Hypertension 1998; 31: 1077-82.

42. Wang YC, Yu CC, Chiu FC et al. Impacts of Mitral E/e' on Myocardial Contractile Motion and Synchronicity in Heart Failure Patients With Reduced Ejection Fraction: An Exercise-Echocardiography Study. Clin Cardiol 2013.

43. Muiesan ML, Salvetti M, Paini A et al. Inappropriate left ventricular mass in patients with primary aldosteronism. Hypertension 2008; 52: 529-34.

44. Kimura G, Uzu T, Nakamura $\mathrm{S}$ et al. High sodium sensitivity and glomerular hypertension/hyperfiltration in primary aldosteronism. J Hypertens 1996; 14: $1463-8$

45. Fu Y, Hall JE, Lu D et al. Aldosterone blunts tubuloglomerular feedback by activating macula densa mineralocorticoid receptors. Hypertension 2012; 59: 599-606. 
46. Palatini P, Mormino P, Dorigatti F et al. Glomerular hyperfiltration predicts the development of microalbuminuria in stage 1 hypertension: the HARVEST. Kidney Int 2006; 70: 578-84.

47. Pontremoli R, Leoncini G, Ravera M et al. Microalbuminuria, cardiovascular, and renal risk in primary hypertension. J Am Soc Nephrol 2002; 13 Suppl 3: S169-72.

48. Koren MJ, Devereux RB, Casale PN et al. Relation of left ventricular mass and geometry to morbidity and mortality in uncomplicated essential hypertension. Ann Intern Med 1991; 114: 345-52.

49. Levy D, Garrison RJ, Savage DD et al. Prognostic implications of echocardiographically determined left ventricular mass in the Framingham Heart Study. N Engl J Med 1990; 322: 1561-6.

50. Sharp AS, Tapp RJ, Thom SA et al. Tissue Doppler E/E' ratio is a powerful predictor of primary cardiac events in a hypertensive population: an ASCOT substudy. Eur Heart J 2010; 31: 747-52.

51. Rodriguez L, Garcia M, Ares M et al. Assessment of mitral annular dynamics during diastole by Doppler tissue imaging: comparison with mitral Doppler inflow in subjects without heart disease and in patients with left ventricular hypertrophy. Am Heart J1996; 131: 982-7.

52. Sechi LA, Di Fabio A, Bazzocchi M et al. Intrarenal hemodynamics in primary aldosteronism before and after treatment. The Journal of clinical endocrinology and metabolism 2009; 94: 1191-7. 